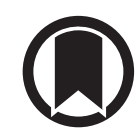

CrossMark

\title{
What kind of emphasis do we need in clinical research to enable personalised respiratory medicine?
}

\author{
To the Editor:
}

The manifesto proposed by RocHe et al. [1] is a most important plan to revolutionise respiratory clinical research.

It also prompts a key question about the balance between the effort spent on randomised clinical trials (RCTs) versus real-life research (RLR), particularly in an era where frequent monitoring of patients capturing their real life, using wearable devices, home diagnostics, smartphones, smart inhalers, collections of contextual information, cloud connectivity and the ability to analyse large complex datasets is becoming increasingly feasible [2].

The promise of the perfect RCT is to provide reliable information on whether the use of a new therapy in a well-defined population of subjects provides a significant benefit versus the same population receiving a control. The fundamental conflict of this approach vis a vis the aspirations of personalised medicine is that the "real-life" subject is unlikely to be anything like the hypothetical "mean" or "median" patient in the RCT, even if that real-life subject were to satisfy all the inclusion/exclusion criteria for the population in the RCT. Furthermore, many RCTs fail to use some form of integrated assessment of efficacy and safety, and therefore do not address the possibility that the "responders" to the therapy may be also experiencing undesirable side-effects. Indeed, this is a deficiency fundamentally inherent in the current regulatory approval process in which the individual benefit/risk ratio is rarely evaluated or used as the primary criterion for approval.

RLR is defined by Roche et al. [1] as "research that includes the widest possible range of the target patients population, cared for in naturalistic conditions, with an intensity of follow-up that does not exceed what is provided in routine care". Such research, if conducted in large numbers of subjects, is eminently suitable to provide the type of information that enables personalised medicine in real-life clinical practice. If the numbers of subjects in such research are sufficiently large to be able to match each patient in the treatment group and control group for many more risk factors than a realistic size RCT would afford, then one can assess prospectively with much greater degree of reliability whether a future subject with the same risk factors is likely to get an attractive benefit/risk ratio from the new intervention. In fact, one could argue that such RLR is more controlled than the traditional RCT, because it controls for many more risk factors! It also removes a key artefact of a typical RCT, which often results in patients in both the treatment and reference groups "feeling better" than they would if they were not in an RCT.

It would seem that much greater investment into RLR associated with collection of the information from such trials into large databases together with analytical tools to extract information from them, will assist in much better decision-making for a personalised approach to respiratory healthcare. This approach may provide a very attractive return on investment for development of new therapies as well and certainly enable their more effective use post-approval through a greater granularity of information about the non-responders and subjects likely to develop side-effects.

While retrospective in its nature, the use of a large registry using "real-life data" enabled KonsTAN et al. [3] to provide evidence of benefits from the chronic use of ibuprofen in well-defined cystic fibrosis patients

@ERSpublications

Real-life clinical research may be more valuable to achieve the aspirations of personalised medicine than the traditional "regulatory style" randomised clinical trials http://bit.ly/2M7vnYT

Cite this article as: Gonda I. What kind of emphasis do we need in clinical research to enable personalised respiratory medicine? Eur Respir J 2020; 55: 1901866 [https://doi.org/10.1183/ 13993003.01866-2019]. 
through propensity matching while also identifying the group that would unlikely derive a positive benefit to risk ratio [3]. This illustrates well the value of RLR-based databases.

Prospective design of such trials can further improve their credibility. RLR can and should be used to create a seamless continuum between therapeutic development and optimisation of the use of therapies post-approval.

Igor Gonda

Respidex LLC, San Francisco, CA, USA.

Correspondence: Igor Gonda, Respidex LLC, PO Box 77565, 460 Brannan Street, San Francisco, CA 94107, USA. E-mail: igonda@respidex.com

Received: 20 Sept 2019 | Accepted: 02 Oct 2019

Conflict of interest: I. Gonda was, until February 2018, the President and CEO of Aradigm Corporation, which has been developing a new treatment for bronchiectasis patients; it was the recognition of the heterogeneity of these patients and the consequent differences in their response to the new therapies that prompted these thoughts about new ways to conduct drug development and the use of new products in clinical practice.

\section{References}

1 Roche N, Anzueto A, Bosnic Anticevich S, et al. The importance of real-life research in respiratory medicine: manifesto of the Respiratory Effectiveness Group. Eur Respir J 2019; 54: 1901511.

2 Gonda I. Re-inventing inhalers for the digital age. RDD Europe 2019 2019; 1: 67-74.

3 Konstan MW, VanDevanter DR, Sawicki GS, et al. Association of high-dose ibuprofen use, lung function decline, and long-term survival in children with cystic fibrosis. Ann Am Thorac Soc 2018; 15: 485-493.

\section{Connected real-life research, a pillar of P4 medicine}

\section{From the authors:}

We thank I. Gonda for outlining the promises and challenges of real-life research (RLR), following the publication of the Respiratory Effectiveness Group manifesto in the European Respiratory Journal [1]. As outlined in this correspondence, the ultimate goal of RLR is to improve patients' outcomes through more precise decision-making in the current era of personalised medicine. To this aim, RLR provides evidence complementing randomised controlled trials (RCTs), especially exploring benefit-risk-cost ratios in both large and specific subpopulations, while minimising the Hawthorne effect. I. Gonda underlines a particularly important aspect of RLR, i.e. how it can be revolutionised by new technologies. This is especially promising in the respiratory field, where therapy administered with inhalation devices plays a major role, while many chronic conditions are under the influence of environmental conditions that can now be continuously recorded.

As recently reviewed [2], mobile devices can 1) include various passive or active sensors and 2) be used for functional assessments (e.g. 6-min walk test). Digital biomarkers can be derived from collected data and used for digital diagnostics and therapeutics. Information provided by these technologies can inform both RCTs and RLR. In the field of RLR, two applications, among others, are currently being investigated with particular interest: monitoring of adherence to inhaled therapy and inhaler technique, and prediction of respiratory events (mostly, exacerbations of chronic conditions such as asthma and COPD).

@ERSpublications

Assessment of $\mathrm{m}$-Health tools needs to include high-quality real-life research studies. Reciprocally, real-life research can benefit highly from opportunities offered by connected devices.

http://bit.ly/2OK4Fqr

Cite this article as: Roche N, Anzueto A, Bosnic Anticevich S, et al. Connected real-life research, a pillar of P4 medicine Eur Respir J 2020; 55: 1902287 [https://doi.org/10.1183/13993003.02287-2019]. 
Various connected inhalers have been studied: for instance, an electronic audio recording device has allowed "profiling" inhaler use in terms of technique and adherence, both intentional and non-intentional [3]. This should provide better understanding of the determinants and consequences of poor adherence/ technique in real life, allowing identification of potential targets for corrective measures [4]. The knowledge gained from connected devices should also help defining the best device for a given patient based on his/her medical and behavioural features, forming the basis of more precise decision-making $[5,6]$. These data should also be collected in effectiveness studies, as a strong explanatory factor: real-life patients often exhibit poor adherence and poor inhalation technique, which are associated with impaired treatment effects [7]. Conversely, in RCTs patients are selected after careful consideration of their likelihood of behaving properly, while investigators closely monitor and reinforce adherence, and check and correct inhalation technique.

Of note, connected devices can also provide feedback to the patient regarding the use of medications, which can in turn enable him/her to react more efficiently, favouring engagement and participation, as well as disease management [8]. The positive consequences of this opportunity need to be assessed rigorously. Again RCTs and RLR are both necessary here, the limitations of "classical" RCTs being the selected nature of the patient population and the close follow-up (also in the control group), which could minimise differences between groups equipped or not with a connected device. Pragmatic RCTs, a component of RLR, are of high interest in this context. Importantly, the device and application are not the only components that need to be assessed: how they are integrated and embedded in patient care at the individual level and in the organisation of care at large is also crucial and represents a major determinant of success [9]. One major challenge is adherence (of both patients and healthcare professionals) to the behavioural changes that $\mathrm{m}$-Health devices aim at inducing [2].

In the field of predictive respiratory medicine, there is a huge variety of m-Health measurements that could inform future studies. They include environmental exposures, medication usage (mentioned above), physiological variables, and so on. Integrating data from these multiple sources (using "classical" biostatistical models or artificial intelligence) will allow potentially useful predictive models to be built with a greater precision. Since $\mathrm{m}$-Health applications can inform patients regarding environmental conditions, they can allow them to avoid areas or conducts at high-risk (due, for example, to atmospheric air pollution or allergen levels). Thus, such applications can be used for prevention of respiratory events, which will also be enriched by the development of predictive models, as mentioned above. Again, the benefit from "preventive m-Health applications" will need to be evaluated in properly designed studies, for which a real-life component will be mandatory. Ultimately, as elegantly portrayed in the book "Deep Medicine" by Eric Topol, the promise of combining all sorts of biosensors with electronic health records, tele-medicine and big data is alluring. Opposite to what many people fear, it has the potential to make medicine more humane, allowing more quality time in all doctor-patient engagements [10].

In conclusion, m-Health devices and applications, including connected inhalation devices, provide tools that can serve $\mathrm{P} 4$ medicine (predictive, preventive, personalised and participatory), favouring treatment personalisation, patient participation, and prediction and prevention of unwanted events. To reach these goals, m-Health tools need to be well integrated in the global organisation of care and rigorously assessed though adequate research studies. In that context, RLR is absolutely necessary to account for all real-life effect modifiers (confounders) and to allow reliable conclusions to be drawn regarding the benefits that can be expected for patients and society.

Nicolas Roche ${ }^{1,2}$, Antonio Anzueto ${ }^{2,3}$, Sinthia Bosnic Anticevich ${ }^{2,4}$, Alan Kaplan ${ }^{2,5}$, Marc Miravitlles $\circledast^{2,6}$, Dermot Ryan $\oplus^{2,7}$, Joan B. Soriano $\circledast^{2,8}$, Omar Usmani ${ }^{2,9}$, Nikos Papadopoulos $\circledast^{2,10,11}$ and G. Walter Canonica ${ }^{2,12}$ ${ }^{1}$ Respiratory Medicine, Hôpitaux Universitaires Paris Centre, Hôpital Cochin, AP-HP and Université de Paris (UMR1016), Paris, France. ${ }^{2}$ Respiratory Effectiveness Group, Board of Directors, Ely, UK. ${ }^{3}$ University of Texas Health and South Texas Veterans Health Care System, San Antonio, TX, USA. ${ }^{4}$ Woolcock Institute of Medical Research, The University of Sydney, Sydney Local Health District, Sydney, Australia. ${ }^{5}$ Dept of Family and Community Medicine, University of Toronto, Toronto, ON, Canada. ${ }^{6}$ Pneumology Dept, University Hospital Vall d'Hebron, Ciber de Enfermedades Respiratorias (CIBERES), Barcelona, Spain. ${ }^{7}$ Allergy and Respiratory Research Group, Usher Institute of Population Health Sciences and Informatics, University of Edinburgh, Medical School, Edinburgh, UK. ${ }^{8}$ Hospital Universitario de la Princesa, Universidad Autónoma de Madrid and Centro de Investigación en Red de Enfermedades Respiratorias (CIBERES), Instituto de Salud Carlos III (ISCIII), Madrid, Spain. ${ }^{9}$ National Heart and Lung Institute, Imperial College London and Royal Brompton Hospital, Airways Disease Section, London, UK. ${ }^{10}$ Division of Infection, Immunity and Respiratory Medicine, University of Manchester, Manchester, UK. ${ }^{11}$ Allergy Dept, 2nd Pediatric Clinic, National and Kapodistrian University of Athens, Athens, Greece. ${ }^{12}$ Respiratory Disease and Allergy Clinic, IRCCS Humanitas Clinical and Research Center, Humanitas University, Milan, Italy.

Correspondence: Nicolas Roche, Pneumologie, Hôpital Cochin, 27, rue du Fbg St Jacques, 75014 Paris, France.

E-mail: nicolas.roche@aphp.fr

Received: 26 Nov 2019 | Accepted: 27 Nov 2019 
Conflict of interest: N. Roche reports grants and personal fees from Boehringer Ingelheim, Novartis and Pfizer, personal fees from Teva, GSK, AstraZeneca, Chiesi, Mundipharma, Sanofi, Sandoz, 3M, Zambon and Trudell, outside the submitted work. A. Anzueto reports personal fees for consultancy from GlaxoSmithKline, AstraZeneca and Boehringer Ingelheim, outside the submitted work. S. Bosnic Anticevich reports personal fees for advisory board work from TEVA, personal fees for consultancy from GSK and MEDA, grants from TEVA, personal fees for lectures from TEVA, GSK and AstraZeneca, personal fees for manuscript preparation from MEDA, personal fees for educational activities from GSK, outside the submitted work. A. Kaplan reports grants and personal fees from AstraZeneca, Boehringer Ingelheim and Pfizer, personal fees from Covis, GSK, Sanofi, Merck Frosst, Novartis, Purdue, Teva, Trudel, Novo Nordisk and Griffols, during the conduct of the study. M. Miravitlles reports personal fees for lectures from AstraZeneca, Boehringer Ingelheim, Chiesi, Cipla, Menarini, Rovi, Bial, Zambon, CSL Behring, Grifols and Novartis, personal fees for consultancy from AstraZeneca, Boehringer Ingelheim, Chiesi, GlaxoSmithKline, Bial, Gebro Pharma, CSL Behring, Laboratorios Esteve, Ferrer, Mereo Biopharma, Verona Pharma, TEVA, pH Pharma, Novartis and Grifols, grants from GlaxoSmithKline and Grifols, outside the submitted work. D. Ryan reports personal fees for advisory board work from GSK, BI, AZ and Novartis, personal fees for lectures from AZ and MEDA, grants meeting registration from MEDA, outside the submitted work; and is Previous President and Current Vice President of REG, and Consultant Strategic Medical Adviser, Optimum Patient Care. J.B. Soriano participated in speaking activities, advisory committees and consultancies during the period 2014-2019 sponsored by: Almirall, AstraZeneca, Boehringer Ingelheim, CHEST, Chiesi, ERS, GEBRO, Grifols, GSK, Linde, Lipopharma, Mundipharma, Novartis, Pfizer, RiRL, Rovi, Sandoz, SEPAR and Takeda; J.B. Soriano declares not receiving ever, directly or indirectly, funding from the tobacco industry or its affiliates. O. Usmani reports grants and personal fees from AstraZeneca, Boehringer Ingelheim and Chiesi, personal fees from Aerocrine, Napp, Mundipharma, Sandoz, Takeda, Zentiva and Cipla, grants from GlaxoSmithKline and Edmond Pharma, outside the submitted work. N. Papadopoulos reports personal fees for advisory board work and lectures from Novartis, Nutricia, HAL, MENARINI/FAES FARMA and MYLAN/MEDA, personal fees for lectures from SANOFI, BIOMAY, MSD, ASIT BIOTECH and Boehringer Ingelheim, personal fees for advisory board work from AstraZeneca and GSK, grants from Gerolymatos International SA and Capricare, outside the submitted work. G.W. Canonica has nothing to disclose.

\section{References}

1 Roche N, Anzueto A, Bosnic Anticevich S, et al. The importance of real-life research in respiratory medicine: manifesto of the Respiratory Effectiveness Group. Eur Respir J 2019; 54: 1901511.

2 Sim I. Mobile devices and health. N Engl J Med 2019; 381: 956-968.

3 Sulaiman I, Cushen B, Greene G, et al. Objective assessment of adherence to inhalers by patients with chronic obstructive pulmonary disease. Am J Respir Crit Care Med 2017; 195: 1333-1343.

4 Blakey JD, Bender BG, Dima AL, et al. Digital technologies and adherence in respiratory diseases: the road ahead. Eur Respir J 2018; 52: 1801147.

5 Dekhuijzen PNR, Vincken W, Virchow JC, et al. Prescription of inhalers in asthma and COPD: towards a rational, rapid and effective approach. Respir Med 2013; 107: 1817-1821.

6 Chung KF. Personalised medicine in asthma: time for action. Eur Respir Rev 2017; 26: 170064.

7 Giraud V, Allaert F-A, Roche N. Inhaler technique and asthma: feasibility and acceptability of training by pharmacists. Respir Med 2011; 105: 1815-1822.

8 Bittner B, Schmit Chiesi C, Kharawala S, et al. Connected drug delivery devices to complement drug treatments: potential to facilitate disease management in home setting. Med Devices (Auckl) 2019; 12: 101-127.

9 Bonini M, Usmani OS. Novel methods for device and adherence monitoring in asthma. Curr Opin Pulm Med 2018; 24: 63-69.

10 Topol E. Deep Medicine: How Artificial Intelligence Can Make Healthcare Human Again. New York, Basic Books, 2019. 